\title{
GENERALIZED GREEN'S FUNCTIONS FOR HIGHER ORDER BOUNDARY VALUE MATRIX DIFFERENTIAL SYSTEMS
}

\author{
R. J. VILLANUEVA and L. JODAR \\ Departamento de Matemática Aplicada. \\ Universidad Politécnica de Valencla. \\ P.O.Box 22012, Valencla, SPAIN. \\ (Received May 29, 1991)
}

ABSTRACT. In this paper, a Green's matrix function for higher order two point boundary value differential matrix problems is constructed. By using the concept of rectangular co-solution of certain algebraic matrix equation associated to the problem, an existence condition as well as an explicit closed form expression for the solution of possibly not well-posed boundary value problems is given avoiding the increase of the problem dimension.

KEYWORDS AND PHRASES. Two point boundary value problem, Green's matrix function, co-solution, algebraic matrix equation, Moore-Penrose pseudoinverse.

CLASSIFICATION A.M.S. (1991). 34B27 15A24 34B10 $34 \mathrm{~B} 05$.

\section{1. - INTRODUCTION.}

Two-point boundary value problems for higher order matrix of differential systems of the type

$$
\begin{aligned}
& X^{(p)}+A_{p-1} X^{(p-1)}+\ldots+A_{1} X^{(1)}+A_{0} X=f(t) ; \quad 0 \leq t \leq b \\
& \sum_{j=1}^{p}\left\{E_{1 j} X^{(j-1)}(0)+F_{1 j} X^{(j-1)}(b)\right\}=r_{1} ; \quad 1 \leq 1 \leq q .
\end{aligned}
$$

where $f(t), X(t), r_{1}$ are matrices in $c^{n \times m}$ for $1 \leq i \leq q$ and $A_{k}, E_{1 j}, F_{1}$, are matrices in $c^{n \times n}$ for $1 \leq i \leq q, 1 \leq j \leq p, 0 \leq k \leq p-1$, appear in different physical problems [1, chap 1].

The standard approach to study such problems is based on the consideration of an extended first order problem

$$
Y^{\prime}(t)=C Y(t)+F(t) ; B_{a} Y(a)+B_{b} Y(b)=R
$$

where $Y=\left(X, X^{\prime}, \ldots, X^{(p-1)}\right)^{T}, F=(0, \ldots, f)^{T}$ are matrices in $C^{n p x m} B_{a}$ and $B_{b}$ are appropriate matrices in $\mathbf{C}^{\text {nqxnp }}, R$ is a matrix in $\mathbf{C}^{\text {nqxn }}$, and $C$ is the companion matrix defined by 


$$
C=\left[\begin{array}{cccc}
0 & I & \cdots & 0 \\
\cdot & & \cdot & \cdot \\
\cdot & & \cdot & \cdot \\
\cdot & & & \cdot \\
\cdot & & & I \\
-A_{0}-A_{1} & \cdots & -A_{p-1}
\end{array}\right]
$$

See [1][3] [14].

This classical approach has the inconvenience of the lack of explicitness due to the relationship $X(t)=[1,0, \ldots, 0] Y(t)$, as well as the computational cost due to the increase of the problem dimension. In particular it needs the computation of the matrix exponential $\exp (t C)$ and it is well known that it is not an easy task [11].

These inconveniences motivates the study of some alternative approach that avoids the increase of the problem dimension. In [4], a solution for a very particular second order problem of the type (1.1) is proposed avoiding the increase of the problem dimension, however, the method is not applicable to more general problems. In a recent paper [7] a method for solving problems of the type (1.1) for the case $p=2$, without considering the extended system (1.2) have been proposed. Results of [7] are based on the existence of an appropriate pair of solutions of the characteristic algebraic matrix equation

$$
Z^{2}+A_{1} Z+A_{0}=0
$$

Unfortunately, equation (1.4) may be unsolvable [6] and in such case, the method given in [7] is not available.

The aim of this paper is to study an existence condition for the solution of problem (1.1) as well as an explicit expression of a solution of the problem in terms of a generalized Green's matrix function $G(t, s)$, taking advantage of the ideas developed in [7] but without the restriction of the existence of solutions of the associated algebraic matrix equation

$$
Z^{p}+A_{p-1} Z^{p-1}+\ldots+A_{1} Z+A_{0}=0
$$

The paper is organized as follows. In section 2, we introduce the concept of rectangular co-solution for the equation (1.5) and we state some results recently given [8], that will be used in the following sections. In section 3 , we construct a generalized Green's matrix function of problem (1.1) by using an appropriate set of co-solutions of equation (1.5) and a procedure analogous to the one developed in [5] for the scalar case. Finally, in section 4 an explicit closed form solution of problem (1.1) in terms of a generalized Green's matrix function is given.

If $S$ is a matrix in $C^{m x n}$, we denote by $S^{+}$its Moore-Penrose pseudoinverse. We recall that an account of uses and properties of this concept may be found in [2] and that the computation of $\mathrm{S}^{+}$is an easy matter using MATLAB [10]. 


\section{2. - RECTANGULAR CO-SOLUTIONS OF POLYNOMIAL MATRIX EQUATIONS AND APPLICATIONS.}

We begin by introducing the concept of rectangular co-solution of equation (1.5), recently given in [8].

DEFINITION 2.1. We say that $(X, T)$ is a $(n, q)$ co-solution of equation (1.5) if $X$ $\in \mathbb{C}^{n \times q}, T \in \mathbb{C}^{q \times q}, X \neq 0$ and

$$
X T^{p}+A_{p-1} X T^{p-1}+\ldots+A_{0} X=0
$$

DEFINITION 2.2. Let $\left(X_{1}, T_{1}\right)$ be a $\left(n, m_{1}\right)$ co-solution for $1 \leq i \leq k$. We say that $\left\{\left(X_{1}, T_{1}\right), 1 \leq i \leq k\right\}$ is a k-complete set of co-solutions of (1.5) if the block matrix $W=\left(W_{1 j}\right)$, with $w_{1 j}=x_{j} T_{j}^{1-1}$ for $1 \leq i \leq p, 1 \leq j \leq k$, is invertible.

THEOREM 1. ( $[8])$ Let $C$ be the companion matrix. If $M=\left(M_{1}\right)$ with $M_{1}, \in C^{n \times m}$, is a nonsingular matrix in $C^{\text {npxnp }}, 1 \leq i \leq p, 1 \leq j \leq k$, and if the Jordan canonical form $J$ of $C$ is $J=\operatorname{diag}\left(J_{1}, \ldots, J_{k}\right)$, with $J, \in C^{m}, m_{j}, m_{1}+\ldots+m_{k}=n p$, such that

$$
M \operatorname{diag}\left(J_{1}, \ldots, J_{k}\right)=C M
$$

then $\left\{\left(M_{1 s}, J_{s}\right), 1 \leq s \leq k\right\}$ is a $k$-complete set of co-solutions of (1.5).

COROLLARY 1.([8]) Let us suppose the notation of theorem 1 , and $\operatorname{let}\left\{\left(\mathrm{M}_{18}, \mathrm{~J}_{\mathrm{s}}\right)\right.$, $1 \leq s \leq k\}$ be a $k$-complete set of co-solutions of equation (1.5). Then, the general solution of the matrix differential equation (1.1) is given by

$$
X(t)=\sum_{s=1}^{k} M_{1 s} \exp \left(t J_{s}\right) D_{s}
$$

where $D_{s}$, is an arbitrary matrix in $C_{s}{ }_{s}$. If $W$ is the block partitioned matrix associated to the set $\left\{\left(M_{18}, J_{s}\right), 1 \leq s \leq k\right\}$ by definition 2.2 , the only solution of (1.1) satisfying the Cauchy conditions $x^{(j)}(0)=c_{j}, 0 \leq j \leq p-1$, is given by (2.3), where the matrices $D_{s}$, for $1 \leq i \leq k$, are uniquely determined by the expression

$$
\left[\begin{array}{c}
\mathrm{D}_{1} \\
\vdots \\
\mathrm{D}_{k}
\end{array}\right]=W^{-1}\left[\begin{array}{l}
\mathrm{C}_{0} \\
\vdots \\
\mathrm{C}_{\mathrm{p}-1}
\end{array}\right] \text {. }
$$

For the sake of clarity in the presentation, we recall a result about the solutions of rectangular systems of equations, that will be used in the following sections.

THEOREM 2. ([13,p.24]) The matrix system $S P=Q$, where $S, P, Q$ are matrices in $\mathbf{C}^{m \times n}, \mathbf{C}^{\mathrm{nxr}}$ and $\mathbf{c}^{\mathrm{m \times r}}$ respectively, is compatible if and only if $S S^{+} Q=Q$ and in this case, the solution of the system is given by

$$
P=S^{+} Q+\left(I-S^{+} S\right) Z,
$$

where $Z$ is an arbitrary matrix in $\mathbf{c}^{\mathrm{nxr}}$. 
Note that under the conditions of theorem 2, a particular solution of system $\mathrm{SP}=\mathrm{Q}$ is given by $\mathrm{P}=\mathrm{S}^{+} \mathrm{Q}$.

\section{3. - CONSTRUCTION OF GREEN'S MATRIX FUNCTIONS.}

Let us consider the homogeneous problem

$$
\begin{array}{ll}
X^{(p)}+A_{p-1} X^{(p-1)}+\ldots+A_{1} X^{(1)}+A_{0} X=0 ; & 0 \leq t \leq b \\
\sum_{j=1}^{R}\left\{E_{1 j} X^{(j-1)}(0)+F_{1 j} X^{(j-1)}(b)\right\}=0 ; & 1 \leq 1 \leq q .
\end{array}
$$

and let $\left\{\left(M_{11}, J_{1}\right), 1 \leq i \leq k\right\}$ be the $k$-complete set of co-solutions of equation (1.5) provided by corollary 1. Then, the general solution of equation (3.1) is given by

$$
x(t)=\sum_{1=1}^{k} U_{1}(t) D_{1}
$$

where $D_{1}$ is an arbitrary matrix in $C^{m_{1}} \times m$ and

$$
\begin{aligned}
U_{1}(t) & =M_{11} \exp \left(t J_{1}\right) . \\
i & =1, \ldots, k .
\end{aligned}
$$

Let us consider the matrix function $G(t, s)$ defined by

$$
G(t, s)= \begin{cases}\sum_{1=1}^{k} U_{1}(t) P_{1}(s), & 0 \leq t \leq s \\ \sum_{i=1}^{k} U_{1}(t) Q_{1}(s), & s \leq t \leq b\end{cases}
$$

where the $C^{m}{ }^{x m}$ valued matrix functions $P_{1}(s), Q_{1}(s)$ have to be determined so that

1.- $G(t, s)$ is a continuous matrix function in $[0, b] \times[0, b]$ and moreover, $\partial^{(j)} \mathrm{G} / \partial \mathrm{t}^{(j)}$ is a continuous function in $(t, s)$, for $(t, s)$ in the triangles $0 \leq t<s \leq b$ and $0 \leq s<t \leq b$ for $j=1, \ldots, p-2$.

2.- If I is the identity matrix in $\mathbf{c}^{\mathrm{n} \times \mathrm{n}}$, one gets the jump discontinuity

$$
\frac{\partial^{(p-1)} G}{\partial t^{(p-1)}}(s+0, s)-\frac{\partial^{(p-1)} G}{\partial t^{(p-1)}}(s-0, s)=I .
$$

3. - As a function of $t, G(t, s)$ satisfies (3.1) and (3.2) in $[0, b]$, if $t \neq s$.

From (3.5) the continuity condition at $t=s$ of Green's function gives us that

$$
\sum_{1=1}^{k} U_{1}(s) P_{1}(s)=\sum_{1=1}^{k} U_{1}(s) Q_{1}(s),
$$


or

$$
\sum_{i=1}^{k} U_{1}(s)\left(P_{1}(s)-Q_{1}(s)\right)=0
$$

On the other hand, by the continuity condition of the partial derivatives of the Green's function until order $p-2$ at $t=s$, we obtain

$$
\sum_{i=1}^{k} U_{i}^{(j)}(s) P_{i}(s)=\sum_{i=1}^{k} U_{i}^{(j)}(s) Q_{1}(s), j=1, \ldots, p-2
$$

and then

$$
\sum_{i=1}^{k} U_{1}^{(j)}(s)\left(P_{1}(s)-Q_{1}(s)\right)=0, j=1, \ldots, p-2
$$

From (3.5) and (3.6) it follows that

$$
\sum_{i=1}^{k} U_{1}^{(p-1)}(s)\left(P_{1}(s)-Q_{1}(s)\right)=I
$$

Let us write

$$
R_{1}(s)=P_{1}(s)-Q_{1}(s), \quad i=1, \ldots, k
$$

then, conditions (3.7) - (3.10) may be written in the compact form

where

$$
U(s)\left[\begin{array}{c}
R_{1}(s) \\
\cdot \\
\cdot \\
\cdot \\
R_{k}(s)
\end{array}\right]=\left[\begin{array}{c}
0 \\
\cdot \\
\dot{0} \\
I
\end{array}\right]
$$

$$
U(s)=\left[\begin{array}{cccc}
U_{1}(s) & \ldots & \ldots & U_{k}(s) \\
\cdot & & & \cdot \\
\cdot & & \cdot \\
\cdot & & \cdot \\
U_{1}^{(p-1)}(s) & \ldots & U_{k}^{(p-1)}(s)
\end{array}\right]
$$

Note that the matrix function $U(s)$ defined by (3.12) is invertible for all $s$ because we may decompose $U(s)$ in the form

$$
U(s)=W \operatorname{diag}\left\{\exp \left(s J_{1}\right), 1 \leq i \leq k\right\}
$$

where

$$
W=\left[\begin{array}{cccc}
M_{11} & \cdots & M_{1 k} \\
\cdot & & & \cdot \\
\cdot & & & \cdot \\
\cdot & & & \cdot \\
M_{11} J_{1}^{p-1} & \ldots & M_{1 k} J_{k}^{p-1}
\end{array}\right]
$$


is invertible since $\left\{\left(M_{1 i}, J_{i}\right), 1 \leq i \leq k\right\}$ is a $k$-complete set of co-solution of equation (1.5).

Let us denote $Y=\left[Y_{1,1 \leq 1 \leq p, 1 \leq j \leq k}\right.$ with $Y_{1,} \in \mathbb{C}_{1}^{m_{1}}$ the inverse of the matrix W. Then, from (3.11) and (3.13), it follows that

$$
R_{1}(s)=\exp \left(-s J_{1}\right) Y_{1 k} ; 1 \leq i \leq k
$$

If we impose that $G(t, s)$ defined by (3.5) satisfies the initial conditions (3.2), we obtain

$$
\begin{gathered}
\sum_{j=1}^{p}\left\{E_{1}, \sum_{m=1}^{k} U_{m}^{(j-1)}(0) P_{m}(s)+F_{1}, \sum_{m=1}^{k} U_{m}^{(j-1)}(b) Q_{m}(s)\right\}=0 \\
1=1, \ldots, q
\end{gathered}
$$

From (3.10) we have

$$
Q_{1}(s)=P_{1}(s)-R_{1}(s), \quad i=1, \ldots, k
$$

Substituting (3.17) into (3.16), it follows that

$$
\begin{gathered}
\sum_{j=1}^{p}\left\{E_{1}, \sum_{m=1}^{k} U_{m}^{(j-1)}(0) P_{m}(s)+F_{1}, \sum_{m=1}^{k} U_{m-1}^{(j-1)}(b)\left[P_{m}(s)-R_{m}(s)\right]\right\}=0 \\
1=1, \ldots, q
\end{gathered}
$$

and

$$
\begin{aligned}
& \sum_{m=1}^{k} \sum_{j=1}^{R}\left(E_{1}, U_{m-1}^{(j-1)}(0)+F_{1 j} U_{m}^{(j-1)}(b)\right) P_{m}(s)= \\
= & \sum_{m=1}^{k} \sum_{j=1}^{R} F_{1 j} U_{m}^{(j-1)}(b) R_{m}(s), \quad 1=1, \ldots, q
\end{aligned}
$$

Let $\mathrm{S}$ be the block matrix

$$
S=\left[\sum_{j=1}^{R}\left(E_{1}, U^{(j-1)}(0)+F_{1 j} U^{(j-1)}(b)\right)\right]_{1 \leq_{1} \leq_{q, 1} S_{a} \leq_{k}}
$$

and let $\mathrm{S}^{+}$be the Moore-Penrose pseudoinverse matrix

$$
S^{+}=\left[T_{11}\right]_{1 \leq m \leq k, 1 \leq 1 \leq q} \text {, with } T_{m i} \in C_{1}^{m_{1} \times n}
$$


Note that the conditions (3.18) may be written in the form

$$
S\left[\begin{array}{c}
P_{1}(s) \\
\cdot \\
\cdot \\
\cdot \\
P_{k}(s)
\end{array}\right]=\left[\begin{array}{c}
\sum_{m=1}^{k} \sum_{j=1}^{P} F_{1 j} U^{(j-1)}(b) R_{m}(s) \\
\cdot \\
\sum_{m=1}^{k} \sum_{j=1}^{P} F_{q j} U_{m}^{(j-1)}(b) R_{m}(s)
\end{array}\right]
$$

From theorem 2, the equation (3.21) is solvable if and only if

$$
S S^{+}\left[\begin{array}{c}
\sum_{m=1}^{k} \sum_{j=1}^{R} F_{1 j} U_{m}^{(j-1)}(b) R_{m}(s) \\
\cdot \\
\sum_{m=1}^{k} \sum_{j=1}^{R} F_{q j} U_{m}^{(j-1)}(b) R_{m}(s)
\end{array}\right]=\left[\begin{array}{c}
\sum_{m=1}^{k} \sum_{j=1}^{R} F_{1 j} U_{m}^{(j-1)}(b) R_{m}(s) \\
\cdot \\
\cdot \\
\sum_{m=1}^{k} \sum_{j=1}^{R} F_{q j} U_{m}^{(j-1)}(b) R_{m}(s)
\end{array}\right]
$$

Let us suppose the algebraic equation (3.21) is compatible. Then, from theorem 2 and (3.20) a solution of (3.21) is given by

$$
\left[\begin{array}{c}
P_{1}(s) \\
\cdot \\
\cdot \\
P_{k}(s)
\end{array}\right]=\left[\begin{array}{ccc}
T_{11} & \cdots & T_{1 q} \\
\cdot & & \cdot \\
\cdot & & \cdot \\
\cdot & & \cdot \\
T_{k 1} & \cdots & T_{k q}
\end{array}\right]\left[\begin{array}{c}
\sum_{j=1}^{R} \sum_{m=1}^{k} F_{1 j} U_{m p}^{(j-1)}(b) \exp \left(-s J_{m}\right) Y_{m p} \\
\cdot \\
\sum_{j=1}^{R} \sum_{m=1}^{k} F_{q j} U^{(j-1)}(b) \exp \left(-s J_{m}\right) Y_{m p}
\end{array}\right]
$$

and then,

$$
\begin{aligned}
& \qquad \begin{array}{l}
P_{1}(s)=\sum_{1=1} \sum_{j=1}^{R} \sum_{m=1}^{k} T_{1}, F_{1}, U_{m}^{(j-1)}(b) \exp \left(-s J_{m}\right) Y_{m p} \\
1=1, \ldots, k \text {. }
\end{array} \\
& \text { Hence and from (3.15), (3.17) it follows that }
\end{aligned}
$$

$$
\begin{gathered}
Q_{1}(s)=P_{1}(s)-R_{1}(s)= \\
=\left(\sum_{1=1}^{R} \sum_{j=1}^{R} \sum_{m=1}^{k} T_{11} F_{1}, U_{m-1)}^{(j-1)} \exp \left(-s J_{m}\right) Y_{m p}\right)-\exp \left(-s J_{1}\right) Y_{1 p} \\
1=1, \ldots, k .
\end{gathered}
$$

Thus the following result has been established

THEOREM 3. Let $\left\{\left(M_{11}, J_{1}\right), 1 \leq i \leq k\right\}$ be the $k$-complete set of co-solutions of equation (1.5) given by theorem 1 and let $\left\{U_{1}(t), 1 \leq i \leq k\right\}$ be defined by (3.4). If condition (3.22) is given, then the boundary value matrix problem (3.1) - (3.2) has a generalized Green's matrix function defined by $(3.5)$, where $P_{1}(s)$ and $Q_{1}(s)$ are given by (3.23) and (3.24). 
REank. If the matrix $S$ has full rank, then, from [2,p.12] $\mathrm{S}^{+} \mathrm{S}=\mathrm{I},(3.21)$ has only one solution and there exists a unique Green's matrix function.

\section{4. - SOLUTION OF THE NON-HOMOGENEOUS BOUNDARY PROBLEM.}

Let us consider the intermediate boundary value problem,

$$
\begin{gathered}
X^{(p)}+A_{p-1} X^{(p-1)}+\ldots+A_{1} X^{(1)}+A_{0} X=f(t) \\
\sum_{j=1}^{p} E_{1}, X^{(j-1)}(0)+F_{1}, X^{(j-1)}(b)=0, \quad 1=1, \ldots, q
\end{gathered}
$$

where $f(t)$ is a $c^{n \times m}$ valued continuous matrix function in $[0, b]$.

Let $x(t)$ be defined by

$$
\begin{gathered}
X(t)=\int_{0}^{b} G(t, s) f(s) d s= \\
=\int_{0}^{t} G(t, s) f(s) d s+\int_{t}^{b} G(t, s) f(s) d s .
\end{gathered}
$$

Taking derivatives and using the Leibniz' rule, we have

$$
\begin{aligned}
& X^{\prime}(t)=\int_{0}^{t} \frac{\partial G(t, s)}{\partial t} f(s) d s+G(t, t) f(t)+\int_{t}^{b} \frac{\partial G(t, s)}{\partial t} f(s) d s- \\
& -G(t, t) f(t)=\int_{0}^{b} \frac{\partial G(t, s)}{\partial t} f(s) d s . \\
& X^{\prime \prime}(t)=\int_{0}^{t} \frac{\partial^{2} G(t, s)}{\partial t^{2}} f(s) d s+\frac{\partial G(t, t)}{\partial t} f(t)+\int_{t}^{b} \frac{\partial^{2} G(t, s)}{\partial t^{2}} f(s) d s- \\
& -\frac{\partial G(t, t)}{\partial t} f(t)=\int_{0}^{b} \frac{\partial^{2} G(t, s)}{\partial t^{2}} f(s) d s . \\
& X^{(p-1)}(t)=\int_{0}^{b} \frac{\partial^{(p-1)} G(t, s)}{\partial t^{(p-1)}} f(s) d s \\
& X^{(p)}(t)=\int_{0}^{t} \frac{\partial^{(p)} G(t, s)}{\partial t^{(p)}} f(s) d s+\frac{\partial^{(p-1)} G(t, t-0)}{\partial t^{(p-1)}} f(t)+ \\
& +\int_{t}^{b} \frac{\partial^{(p)} G(t, s)}{\partial t^{(p)}} f(s) d s-\frac{\partial^{(p-1)} G(t, t+0)}{\partial t^{(p-1)}} f(t)= \\
& =+\int_{0}^{b} \frac{\partial^{(p)} G(t, s)}{\partial t^{(p)}} f(s) d s+\left(\frac{\partial^{(p-1)} G(t, t-0)}{\partial t^{(p-1)}}-\frac{\partial^{(p-1)} G(t, t+0)}{\partial t^{(p-1)}}\right) f(t)= \\
& =\int_{0}^{b} \frac{\partial^{(p)} G(t, s)}{\partial t^{(p)}} f(s) d s+f(t) .
\end{aligned}
$$


Hence and from the properties of $G(t, s)$ it follows that

$$
\begin{gathered}
X^{(p)}+A_{p-1} X^{(p-1)}+\ldots+A_{1} X^{(1)}+A_{0} X= \\
=\int_{0}^{b} \frac{\partial^{(p)} G(t, s)}{\partial t^{(p)}} f(s) d s+f(t)+ \\
+A_{p-1} \int_{0}^{b} \frac{\partial^{(p-1)} G(t, s)}{\partial t^{(p-1)}} f(s) d s+\ldots+A_{0} \int_{0}^{b} G(t, s) f(s) d s= \\
\int_{0}^{b}\left(\frac{\partial^{(p)} G(t, s)}{\partial t^{(p)}}+A_{p-1} \frac{\partial^{(p-1)} G(t, s)}{\partial t^{(p-1)}}+\ldots A_{0} G(t, s)\right. \\
=f(t) .
\end{gathered}
$$

and

$$
\begin{gathered}
\sum_{j=1}^{p}\left\{E_{1 j} X^{(j-1)}(0)+F_{1 j} X^{(j-1)}(b)\right\}= \\
\int_{0}^{b}\left(\sum_{j=1}^{p}\left\{E_{1 j} \frac{\partial^{(j-1)} G(0, s)}{\partial t^{(j-1)}}+F_{1 j} \frac{\partial^{(j-1)} G(b, s)}{\partial t^{(j-1)}}\right\} f(s) d s=0,\right. \\
i=1, \ldots, q .
\end{gathered}
$$

Now let us consider the auxiliary problem

$$
\begin{gathered}
X^{(p)}+A_{p-1} X^{(p-1)}+\ldots+A_{1} X^{(1)}+A_{0} X=0 \\
\sum_{j=1}^{p} E_{1}, X^{(j-1)}(0)+F_{1}, X^{(j-1)}(b)=r_{1}, \\
i=1, \ldots, q .
\end{gathered}
$$

Then, from the corollary 1, the form of the solutions of (4.3) is

$$
X(t)=\sum_{m=1}^{k} U_{m}(t) Q_{m}
$$

The boundary value conditions of (4.4) give us the next expression

or

$$
\begin{gathered}
\sum_{j=1}^{R}\left\{E_{1}, \sum_{m=1}^{k} U_{m}^{(j-1)}(0) Q_{m}+F_{1 j} \sum_{m=1}^{k} U_{m}^{(j-1)}(b) Q_{m}\right\}=r_{1} \\
i=1, \ldots, q .
\end{gathered}
$$

$$
\sum_{j=1}^{R} \sum_{m=1}^{k}\left\{E_{1}, U_{m}^{(j-1)}(0)+F_{1}, U_{m}^{(j-1)}(b)\right\} Q_{m}=r_{1} \text {. }
$$$$
\mathbf{i}=1, \ldots, q \text {. }
$$ 
If we set the last expression in matrix form

$$
S\left[\begin{array}{c}
Q_{1} \\
\cdot \\
\cdot \\
Q_{k}
\end{array}\right]=\left[\begin{array}{c}
r_{1} \\
\cdot \\
r_{1} \\
\cdot \\
r_{q}
\end{array}\right]
$$

From theorem 2 of section 1, under the compatibility condition

$$
S S^{+}\left[\begin{array}{c}
r_{1} \\
\cdot \\
r_{1} \\
\cdot \\
r_{q}
\end{array}\right]=\left[\begin{array}{c}
r_{1} \\
\cdot \\
r_{1} \\
\cdot \\
r_{q}
\end{array}\right]
$$

and taking into account (3.20), a solution of (4.5) is given by

$$
\left[\begin{array}{c}
Q_{1} \\
\cdot \\
\cdot \\
\cdot \\
Q_{k}
\end{array}\right]=\left[\begin{array}{cc}
T_{11} \ldots \ldots T_{1 q} \\
\cdot & \cdot \\
\cdot & \cdot \\
T_{k 1} \ldots \ldots T_{k q}
\end{array}\right]\left[\begin{array}{c}
r_{1} \\
\cdot \\
r_{1} \\
\cdot \\
r_{q}
\end{array}\right]
$$

Thus, $Q_{m}=\sum_{1=1}^{g} T_{m} r_{1}, 1 \leq m \leq k$, and a solution $G(t)$ of (4.3) - (4.4) is given by the next expression

$$
G(t)=\sum_{n=1}^{k} U_{m}(t)\left(\sum_{1=1}^{g} T_{m 1} r_{1}\right)
$$

Thus, the following result has been proved:

THroRy 4. Let $\left\{\left(M_{11}, J_{1}\right), 1 \leq i \leq k\right\}$ be a k-complete set of co-solutions of equation (1.5) and let $\left\{U_{1}(t), 1 \leq i \leq k\right\}$ be defined by (3.4). If the conditions (3.22) and (4.6) are satisfied, 1.e., the algebraic equations (3.21) and (4.5) are compatible, $S$ is defined by (3.19), $S^{+}=\left[T_{m i}\right]_{1 s_{m} s_{k}, 1 s_{1} s_{q}}$ is the Moore-Penrose pseudo-inverse and $f(t)$ is continuous, then the boundary value problem (1.1) has a solution given by

$$
X(t)=\int_{0}^{b} G(t, s) f(s) d s+G(t),
$$

where $G(t)$, is given by $(4.6)$ and $G(t, s)$ is the generalized Green's matrix function constructed by theoren 3 . 
REMARK. It is interesting to recall that the Jordan canonical form of a matrix may be efficiently computed with MACSYMA [9] and the matrix exponential of a Jordan block has a well known expression [12,p.66].

In the next example, we construct a generalized Green's matrix function for a not well-posed houndary value matrix problem.

EXAMPLE. Let us consider the second order differential equation,

$$
\begin{gathered}
X^{\prime}(t)+A_{1} X^{\prime}(t)+A_{0} X(t)=0 \quad t \in[0,1] \\
E_{11} X(0)+F_{11} X(1)=0 \\
E_{22} X^{\prime}(0)+F_{22} X^{\prime}(1)=0
\end{gathered}
$$

and

$$
\begin{gathered}
A_{1}=\left[\begin{array}{rr}
-1 & 1 \\
0 & -2
\end{array}\right], A_{0}=\left[\begin{array}{ll}
0 & 0 \\
0 & 1
\end{array}\right], E_{11}=\left[\begin{array}{ll}
0 & 1 \\
0 & 1
\end{array}\right], F_{11}=\left[\begin{array}{ll}
0 & 0 \\
0 & 1
\end{array}\right], \\
E_{22}=\left[\begin{array}{rr}
0 & 0 \\
-e & e
\end{array}\right], F_{22}=\left[\begin{array}{ll}
0 & 0 \\
0 & 1
\end{array}\right] .
\end{gathered}
$$

Then, the matrices $M, J$ and $M^{-1}$ are given by

$$
M=\left[\begin{array}{r|rrr}
1 & -1 & 1 & -1 \\
0 & 0 & 1 & -1 \\
\hline 0 & -1 & 0 & 0 \\
0 & 0 & 1 & 0
\end{array}\right], \quad J=\left[\begin{array}{l|lll}
0 & 0 & 0 & 0 \\
\hline 0 & 1 & 1 & 0 \\
0 & 0 & 1 & 1 \\
0 & 0 & 0 & 1
\end{array}\right], \quad M^{-1}=\left[\begin{array}{rr|rr}
1 & -1 & -1 & 0 \\
\hline 0 & 0 & -1 & 0 \\
0 & 0 & 0 & 1 \\
0 & -1 & 0 & 1
\end{array}\right]
$$

and

$$
\operatorname{diag}\left[\exp \left(s J_{1}\right)\right]=e^{t}\left[\begin{array}{llll}
1 & 0 & 0 & 0 \\
0 & 1 & t & t^{2} / 2 \\
0 & 0 & 1 & t \\
0 & 0 & 0 & 1
\end{array}\right]
$$

Thus, a complete set of co-solutions is

$$
\left\{\left(\left[\begin{array}{l}
1 \\
0
\end{array}\right], 0\right),\left(\left[\begin{array}{rrr}
-1 & 1 & -1 \\
0 & 1 & -1
\end{array}\right],\left[\begin{array}{lll}
1 & 1 & 0 \\
0 & 1 & 1 \\
0 & 0 & 1
\end{array}\right]\right)\right\},
$$

and we can compute the expressions $U_{1}(t), U_{2}(t)$, theirs derivatives and $R_{1}(t)$, $R_{2}(t)$.

$$
\begin{gathered}
U_{1}(t)=\left[\begin{array}{l}
1 \\
0
\end{array}\right], U_{1}^{\prime}(t)=\left[\begin{array}{l}
0 \\
0
\end{array}\right], \\
U_{2}(t)=e^{t}\left[\begin{array}{ccc}
-1 \cdot & 1-t & -t^{2} / 2+t-1 \\
0 & 1 & t-1
\end{array}\right], U_{2}^{\prime}(t)=e^{t}\left[\begin{array}{ccc}
-1 & -t & -t\}^{2} \\
0 & 1 & t
\end{array}\right], \\
R_{1}(s)=\exp \left(-s J_{1}\right) Y_{12}=[-1,0], \\
R_{2}(s)=\exp \left(-s J_{2}\right) Y_{22}=e^{-s}\left[\begin{array}{cc}
1 & -s+\frac{s^{2}}{2} \\
0 & 1-s \\
0 & 1
\end{array}\right] .
\end{gathered}
$$


From the boundary value conditions (4.9) the corresponding matrix $S$ defined by (3.19) takes the form

$$
S=\left[\begin{array}{cccc}
0 & 0 & 1 & -1 \\
0 & 0 & 1+e & -1 \\
0 & 0 & 0 & 0 \\
0 & e & 0 & e
\end{array}\right]
$$

that clearly is not invertible. Thus problem (4.8)-(4.10) is not well posed, however, the equality

$$
S\left[\begin{array}{ll}
a & b \\
0 & e^{-s} \\
0 & e^{-s}(1-s) \\
0 & e^{-s}(1-s)
\end{array}\right]=e^{1-s}\left[\begin{array}{cc}
0 & 0 \\
0 & 1-s \\
0 & 0 \\
0 & 2-s
\end{array}\right]
$$

means that the corresponding algebraic equation (3.21) is compatible. Then, the Moore-Penrose pseudo-inverse is given by

$$
S^{+}=\left[\begin{array}{cccc}
0 & 0 & 0 & 0 \\
1+e^{-1} & -e^{-1} & 0 & e^{-1} \\
-e^{-1} & e^{-1} & 0 & 0 \\
-1-e^{-1} & e^{-1} & 0 & 0
\end{array}\right]
$$

Therefore, we can obtain $P_{1}(s)$ and $P_{2}(s)$,

$$
\begin{gathered}
P_{1}(s)=[0,0] \\
P_{2}(s)=e^{-s}\left[\begin{array}{cc}
0 & 1 \\
0 & 1-s \\
0 & 1-s
\end{array}\right]
\end{gathered}
$$

and

$$
\begin{gathered}
Q_{1}(s)=[1,0] . \\
Q_{2}(s)=e^{-s}\left[\begin{array}{cc}
1 & 1+s-\frac{s^{2}}{2} \\
0 & 0 \\
0 & -s
\end{array}\right] .
\end{gathered}
$$

Finally, a generalized Green's matrix function of problem (4.8)-(4.10) is given by

$$
G(t, s)=\left\{\begin{array}{cc}
e^{t-s}\left[\begin{array}{cc}
0 & \frac{s t^{2}}{2}+s t-\frac{t^{2}}{2}-t-1 \\
0 & -s t-s+t+1
\end{array}\right] & 0 \leq t<s \\
{\left[\begin{array}{ll}
1 & 0 \\
0 & 0
\end{array}\right]+e^{t-s}\left[\begin{array}{cc}
-1 & \frac{s t^{2}}{2}+\frac{s^{2}}{2}-s-1 \\
0 & -s t
\end{array}\right]} & s<t \leq 1 .
\end{array}\right.
$$


ACKNOULEDGEizNTS. - This paper has been supported by the NATO grant CRG 900040.

\section{REFERENCES.}

1.- U.M. Ascher, R.M.M. Mattheij and R.D. Russell, "Numerical solution of boundary value problems for ordinary differential equations", Prentice Hall Inc., New Jersey, 1988.

2.- S.L. Campbell and C.D. Meyer Jr., "Generalized inverses of linear transformations". Pitman Pubs.Co.,1979.

3. - R. Conti, "Recent trends in the theory of boundary value problems for ordinary differential equations", Boll. Un. Mat. Ital., 22(1967), 135-178.

4.- K.A. Heimes, "Green's functions for linear second order systems", SIAM J. Math. Anal., $9(1978), 207-214$.

5. - E.L. Ince, "Ordinary differential equations", Dover 1957.

6. - L. Jódar, "Explicit solutions for second order operator differential equations with two boundary value conditions", Linear Algebra Appl., 103(1988), 73-86.

7.- L. Jódar, "Boundary value problems and Green's operator functions", Glasnick Matematicky, 24(44)(1989), 507-518.

8. - L. Jódar and E. Navarro, "Rectangular co-solutions of polynomial matrix equations and applications", Applied Maths. Letters, Vol No.2 (1991),13-16.

9. - MACSYMA, MACSYMA Symbolics Inc. , 1989.

10. - C.B. Moler, "Matlab user's guide", Technical report CS81-1, Department of Computer Science, Univ. of Mexico, Albuquerque, 1980.

11. - C. Moler and Van Loan, "Nineteen dubious ways to compute the exponential of a matrix", SIAM Review, 20(1978), 801-836.

12.- J.M. Ortega, "Numerical analysis. A second course", Academic Press, 1972.

13. - C.R. Rao and S.K. Mitra, "Generalized inverse of matrices and Its applications", Wiley, New York 1971.

14. - W.T. Reid, "Ordinary differentlal equations", John-Wiley, New York 1971. 


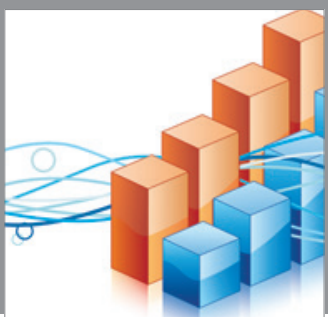

Advances in

Operations Research

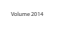

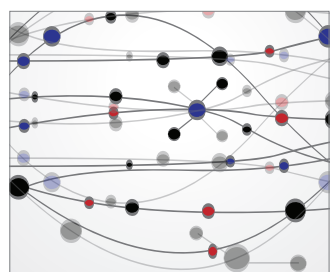

\section{The Scientific} World Journal
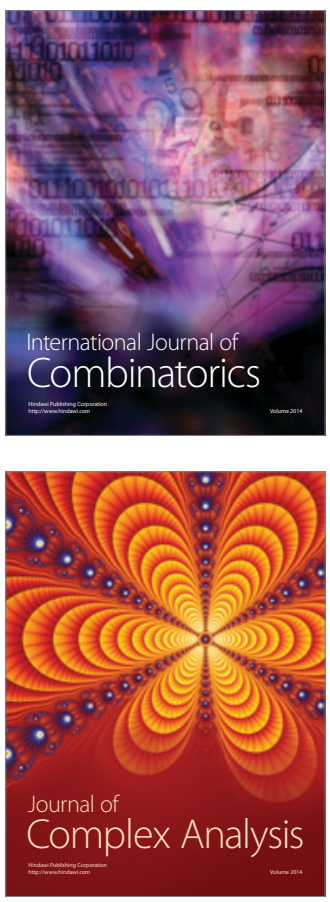

International Journal of

Mathematics and

Mathematical

Sciences
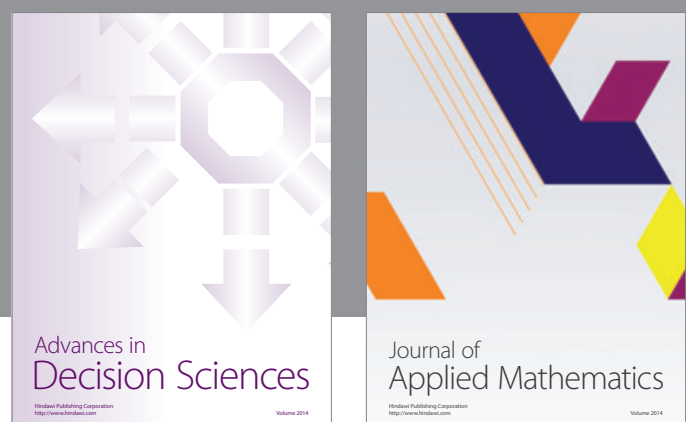

Journal of

Applied Mathematics
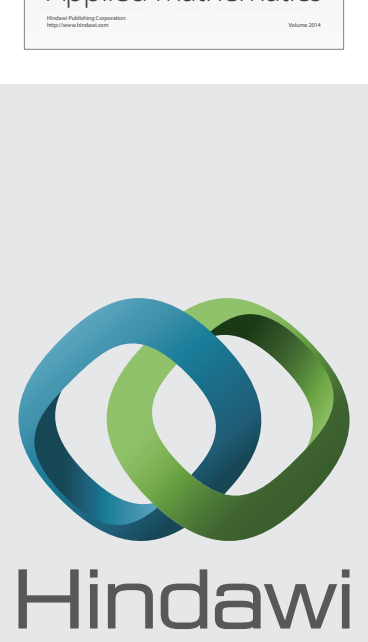

Submit your manuscripts at http://www.hindawi.com
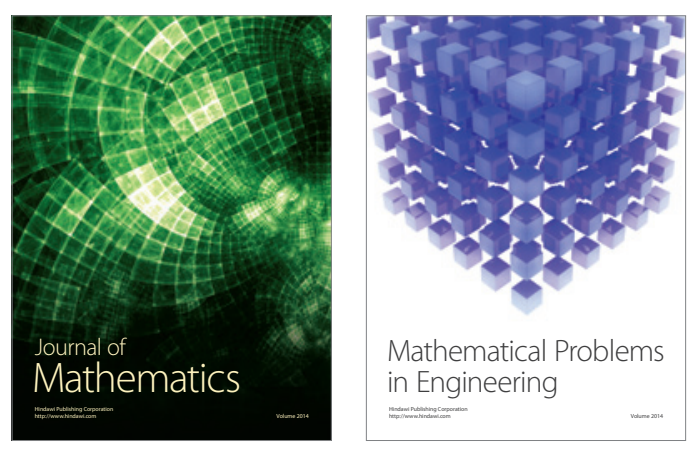

Mathematical Problems in Engineering
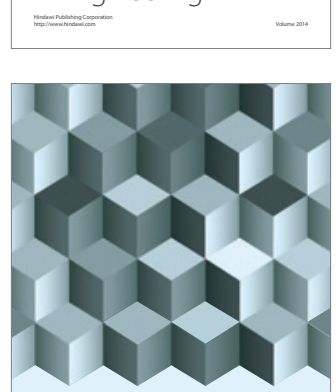

Journal of

Function Spaces
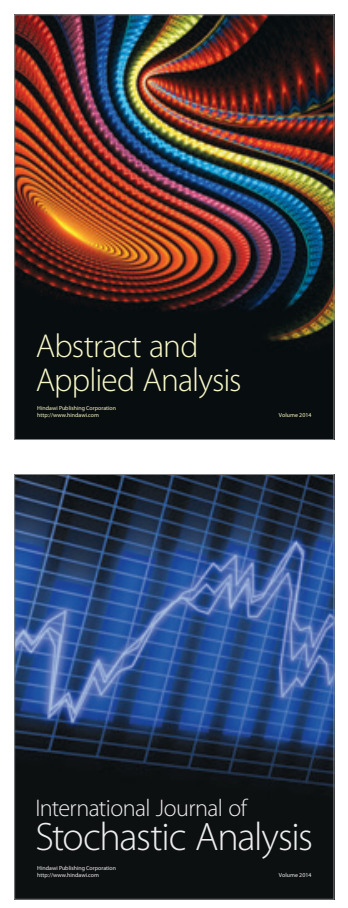

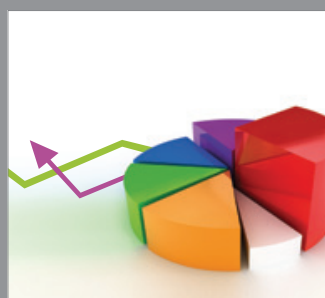

ournal of

Probability and Statistics

Promensencen
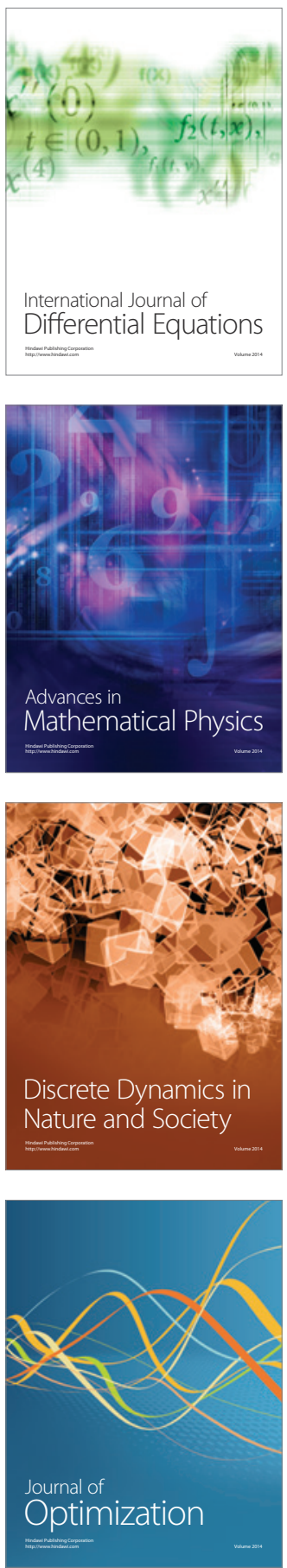